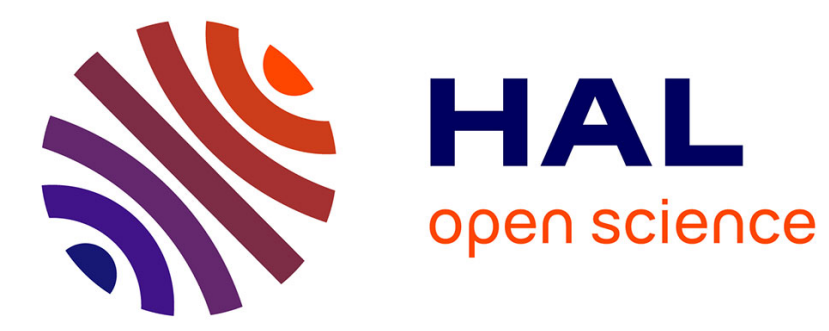

\title{
Exploitation domestique de l'énergie solaire. Influence du climat
}

\author{
J. Fléchon
}

\section{To cite this version:}

J. Fléchon. Exploitation domestique de l'énergie solaire. Influence du climat. Revue de Physique Appliquée, 1978, 13 (2), pp.75-79. 10.1051/rphysap:0197800130207500 . jpa-00244424

\section{HAL Id: jpa-00244424 https://hal.science/jpa-00244424}

Submitted on 1 Jan 1978

HAL is a multi-disciplinary open access archive for the deposit and dissemination of scientific research documents, whether they are published or not. The documents may come from teaching and research institutions in France or abroad, or from public or private research centers.
L'archive ouverte pluridisciplinaire HAL, est destinée au dépôt et à la diffusion de documents scientifiques de niveau recherche, publiés ou non, émanant des établissements d'enseignement et de recherche français ou étrangers, des laboratoires publics ou privés. 


\title{
EXPLOITATION DOMESTIQUE DE L'ÉNERGIE SOLAIRE INFLUENCE DU CLIMAT
}

\author{
J. FLÉCHON \\ Laboratoire de Physique de Dépôts Métalliques, Case Officielle 140, 54037 Nancy Cedex, France
}

(Reçu le 13 juin 1977, révisé le 19 octobre 1977, accepté le 21 octobre 1977)

\begin{abstract}
Résumé. - On fait une étude critique, valable pour la Belgique et l'Est de la France, de l'incidence des conditions climatiques sur l'utilisation domestique de l'énergie solaire.

A partir des enregistrements énergétiques du rayonnement global analysé sur une douzaine d'années on observe une constance remarquable du global moyen journalier par mois. Par contre le global journalier durant un mois quelconque est une grandeur essentiellement fluctuante avec des écarts pouvant dépasser plus de 5 fois la valeur la plus faible. La qualité énergétique du jour peut être appréciée en se fixant sur le global entre $11 \mathrm{~h}$ et $12 \mathrm{~h}$ (TSV).

On examine ensuite les performances possibles d'un capteur plan utilisant l'effet de serre en thermostatique puis en thermocinétique. On évalue enfin les besoins nécessaires au chauffage d'une habitation et on fait le bilan des perspectives ouvertes dans ce domaine par l'emploi du rayonnement solaire.
\end{abstract}

Abstract. - A critical study, valid for Belgium and the East of France, is made of the incidence of climatic conditions on the domestic use of solar energy.

Using energy recordings of global radiation analysed over a period of about twelve years, we observe a remarkable constancy in the global daily average per month. However, the daily global during any one month is of an essentially fluctuating size with the differences able to exceed five times the weakest value. The day's energy quality may be estimated by basing ourselves on the global between 11 and 12 o'clock (TSV).

The possible performances of a plane collector using the greenhouse effect first in thermostatics and then in thermokinetics are next examined. Finally, we evaluate the needs necessary to a home's heating and consider the perspectives opened in this field by the use of solar radiation.

Dans la perspective d'une économie générale des énergies fossiles, l'utilisation du rayonnement solaire au chauffage des habitations et de l'eau nécessaire à la vie domestique est apparue comme une solution élégante et simple destinée à couvrir une partie de nos besoins en calories.

L'absence de pollution, l'apparente gratuité, l'ampleur indéfinie de la source autorisent tous les espoirs surtout si l'on emploie des capteurs fixes sensibles au rayonnement global, d'un fonctionnement autonome, d'une longue fiabilité et d'un entretien quasi négligeable.

Le but de cette communication est de montrer qu'en réalité le problème est complexe, et qu'à la limite, au regard des besoins en Belgique comme dans l'Est de la France, le seul chauffage solaire est insuffisant à la fin de l'automne et au début de l'hiver, il est superflu en été. Le stockage de longue durée reste actuellement un mythe.
1. Données climatiques dans l'Est de la France. Avant d'examiner les performances possibles d'un capteur plan il convient d'observer que le rayonnement solaire global descendant conditionne absolument la quantité et la distribution de la chaleur utilisable et qu'une connaissance précise, étalée sur une longue période, de l'énergie incidente est indispensable. Nous avons retenu le global reçu par un plan horizontal étant donnée la nébulosité de nos régions et le fait que les capteurs plans utilisant l'effet de serre réagissent avcc le global, ce qui n'est pas le cas des concentrateurs. Les stations météorologiques pourvues de pyranomètres font ce travail depuis des années, chaque jour et heure par heure.

Nous avons analysé depuis 1965 les tableaux de nombres traduisant leurs résultats pour les stations de Nancy-Essey et Mâcon. Les 3 paramètres suivants apparaissent : 
1.1 Le Global JOURNALIER MOYEN PAR MOIS. - On peut grouper les mois par 4 pour obtenir :

$\begin{array}{ccccc}\text { Nancy : } & \text { Saison froide } & \text { NDJF } & G \text { moyen } & \\ \text { Demi-saison } & \text { MASO } & <1,5 \\ \text { Saison chaude } \mathrm{kWh} / \mathrm{m}^{2} & 2<G_{\mathrm{m}}<4 \\ \text { MJJA } & & 4,5<G_{\mathrm{m}}<5,5\end{array}$

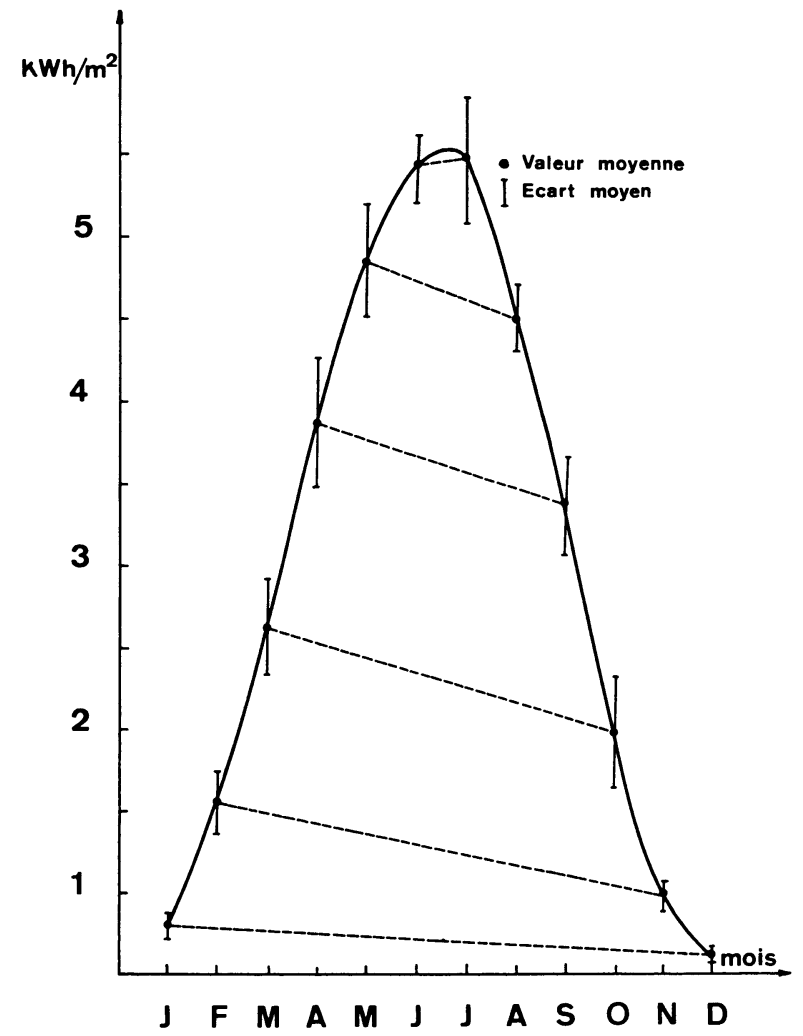

FIG. 1. - Rayonnement solaire global journalier moyen par mois au cours des onze dernières années à Nancy-Essey.

[Daily global solar radiation average by month in the last eleven years at Nancy-Essey.]

La figure 1 résume l'ensemble des observations. Elle porte les écarts moyens à la moyenne : $\Delta G_{\mathrm{m}} / G_{\mathrm{m}}$ varie de 5 à $15 \%$ si l'on tient compte des variations annuelles.

Nous énonçons ce premier résultat essentiel : à environ $10 \%$ près les valeurs journalières moyennes prises par mois : $G_{\mathrm{m}}$ sont constantes. Ainsi l'énergie solaire globale mensuelle reçue au sol dans une région donnée ne varie pas de plus de $\pm 10 \%$ au cours des années.

On peut par exemple se demander si la sécheresse exceptionnelle de 1976 a correspondu à une singularité $\mathrm{du}$ rayonnement. Le tableau I répond négativement à cette question :

\section{TABLEAU I}

$\begin{array}{cccccc}\text { Station } & \text { Mois } & \text { Juin } & \text { Juillet } & \text { Août } & \text { Sept. } \\ - & - & - & - & - & - \\ \text { Nancy- } & \text { Moyenne } & & & & \\ \text { Essey } & (65-75) & 5,5 & 5,5 & 4,5 & 3,5 \\ & 76 & 6,28 & 5,38 & 5,18 & 2,96 \\ \text { Mâcon } & \begin{array}{c}\text { Moyenne } \\ \\ (65-75)\end{array} & 5,9 & 6,05 & 4,96 & 3,78 \\ & 76 & 7,4 & 6,10 & 5,12 & \end{array}$

Par contre une comparaison systématique entre les 2 stations montre la prépondérance de la latitude pour augmenter systématiquement $G_{\mathrm{m}}$ de $10 \%$ au bénéfice de Mâcon.

1.2 Le global journalier à Nancy durant 1 mois quelconque. La figure 2 montre pour le mois de février 1977 les valeurs successives prises chaque jour par $G$. Ce n'est qu'un exemple mais le caractère observé est absolument général.

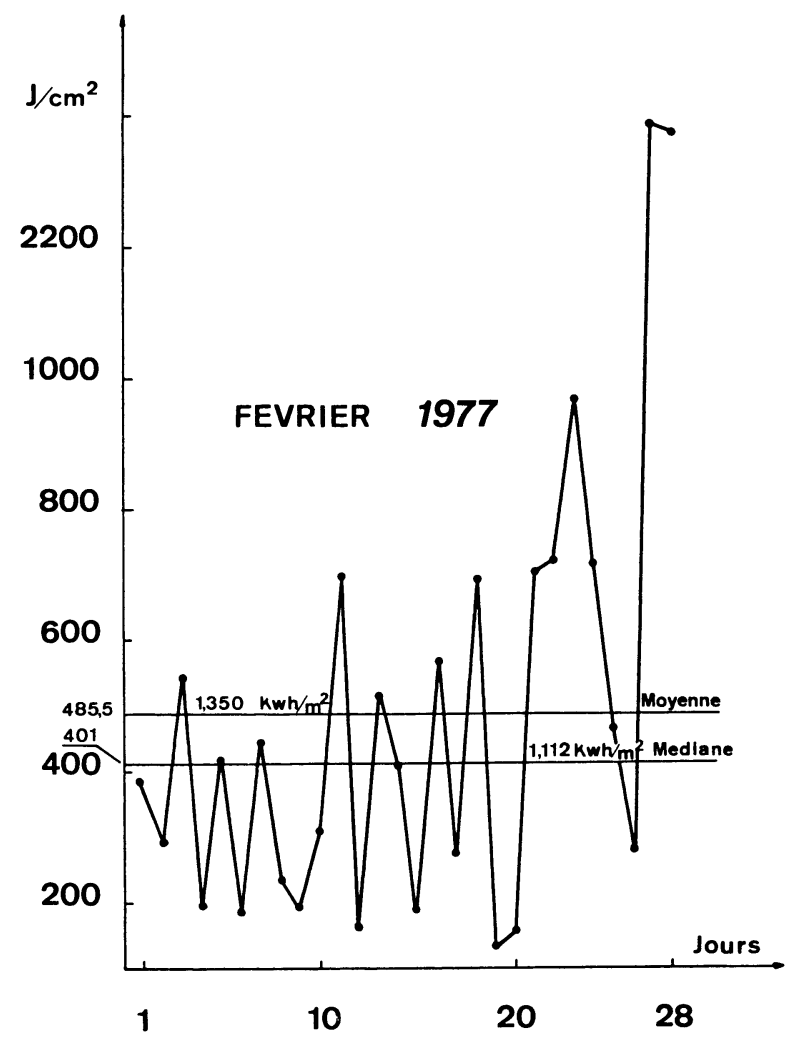

Fig. 2. - Influence de la déclinaison sur l'allure générale du diagramme de la nébulosité sur la place de la médiane.

[Influence of declination on general form of the diagram and of the nebulosity on the place of the mediane.]

Nous énonçons une deuxième proposition, capitale pour l'usage domestique envisagé du rayonnement solaire : si $G_{\mathrm{m}}$ varie peu, $G$ est une grandeur fluctuante, l'écart à $G_{\mathrm{m}}$ pouvant varier de \pm 70 à $\pm 200 \%$, l'écart entre les extrêmes observables durant un mois est susceptible de passer de 4 à 20 fois la valeur la plus faible - les écarts les plus forts se produisent à la saison froide - l'écart entre deux jours consécutifs pouvant atteindre 6 fois la valeur la plus petite.

Notons que si durant une décade les enregistrements de $G$ sont systématiquement inférieurs à $G_{\mathrm{m}}$ il y a ensuite compensation. Par ailleurs, si la nébulosité est particulièrement forte, la médiane s'éloigne de la moyenne. Observons enfin que la variation de la déclinaison solaire se lit sur l'aspect général du diagramme.

1.3 Le global $g$ de $11 \mathrm{~h}$ à $12 \mathrm{~h}$ : Nous avons montré $[1,2]$ en prenant un point de vue différent de celui 
de J. A. Duffie et W. A. Beckman [3] que l'étude thermostatique des insolateurs plans conduit pour un global $g$ donné à une température limite $\theta_{\mathrm{e}} \mathrm{du}$ capteur que des considérations cinétiques [4] présentent comme inaccessible étant donné le temps de relaxation $\tau$ du système.

Nous avons tenté de définir la qualité énergétique journalière en retenant l'énergie $g$ durant l'heure voisine du passage du soleil dans le plan méridien du lieu afin de nous référer à un simple nombre nous renseignant sur la température maximale du fluide caloporteur. Par exemple si

$$
g_{11-12}<100 \mathrm{Wh} / \mathrm{m}^{2}=36 \mathrm{~J} / \mathrm{cm}^{2}
$$

dans une ambiance de $-3^{\circ} \mathrm{C}$ un vent de $10 \mathrm{~m} / \mathrm{s}$ on ne peut espérer dépasser $10^{\circ} \mathrm{C}$ au collecteur. Il suffit alors de compter le nombre de jours qui, en saison froide présentent un apport énergétique particulièrement faible. Nous trouvons ainsi par mois :

\begin{tabular}{lcccc} 
& $\mathrm{N}$ & $\mathrm{D}$ & $\mathrm{J}$ & $\mathrm{F}$ \\
\cline { 2 - 3 } Nancy-Essey & 10 & $\overline{15}$ & $\overline{15}$ & $\overline{3}$ \\
Mâcon & $(7-8)$ & $(10-12)$ & $(10-12)$ & 3
\end{tabular}

Cette constatation est fondamentale. Elle prouve que quelle que soit la qualité du récepteur, à la fin de l'automne et au début de l'hiver près de la moitié des jours correspondent à un déficit énergétique certain, sans être pour autant assuré que l'autre moitié fournira les thermies en suffisance. Fort heureusement il n'en est plus de même en demi-saison.
Ainsi l'examen attentif de l'énergie solaire incidente, de la distribution de son intensité, de ses fluctuations, demeure l'enquête nécessaire avant l'établissement de tout projet de chauffage solaire.

2. Captage d'énergie solaire par l'insolateur plan. Si la connaissance de l'énergie solaire journalière est importante sa valeur maximale ainsi que la durée d'irradiation efficace ne le sont pas moins. En effet deux problèmes se posent :

2.1 Thermostatique. - La température limite $T_{\mathrm{e}}$ du récepteur est liée au global $G$ par une loi de type Stéfan

$$
G=\sigma_{1} T_{\mathrm{e}}^{4}
$$

$\sigma_{1}<\sigma$ : constante de Stéfan. $\sigma_{1}$ que nous appellerons constante thermostatique de l'insolateur caractérise l'effet de serre puisqu'à $G$ donné on peut écrire

$$
\sigma T_{\mathrm{n}}^{4}=\sigma_{1} T_{\mathrm{e}}^{4}
$$

$T_{\mathrm{n}}$ désignant la température d'équilibre du corps noir soumis au rayonnement $G$. Ainsi $T_{\mathrm{e}}>T_{\mathrm{n}}$ à l'équilibre. Nous avons pu établir ce résultat en nous limitant d'abord aux échanges radiatifs [1] puis le généraliser [2] en introduisant le rayonnement terrestre, la convection interne, la convection externe, l'influence $\mathrm{du}$ vent.

Bornons-nous ici à la mise en équation traduisant des bilans d'énergie dans le cas de 2 vitres :

$$
\begin{array}{ll}
\text { collecteur } & G(1-r)^{4}+\sigma T_{1}^{4}=\sigma T_{\mathrm{e}}^{4}+h\left(T_{\mathrm{e}}-T_{1}\right)^{a} \\
1^{\mathrm{re}} \text { vitre (interne) } & \sigma\left(T_{\mathrm{e}}^{4}+T_{2}^{4}\right)+h\left(T_{\mathrm{e}}-T_{1}\right)^{a}=2 \sigma T_{1}^{4}+h\left(T_{1}-T_{2}\right)^{a} \\
2^{\mathrm{e}} \text { vitre (externe) } & \sigma T_{1}^{4}+\varepsilon_{1} \sigma T_{\mathrm{a}}^{4}+h\left(T_{1}-T_{2}\right)^{a}=2 \sigma T_{2}^{4}+10 b\left(T_{2}-T_{\mathrm{a}}\right)(\text { vent } 3 \mathrm{~m} / \mathrm{s})
\end{array}
$$

$G \quad$ : global, $r$ : facteur de réflexion normale d'un plan de verre,

$\sigma \quad$ : constante de Stéfan du collecteur et des vitres assimilées à des corps noirs pour les I. R.,

$T_{1} \quad$ : température d'équilibre de la $1^{\text {re }}$ vitre,

$T_{\mathrm{e}} \quad$ : température d'équilibre du collecteur,

$T_{\mathrm{a}} \quad:$ ambiante,

$h$ et $a$ : constantes définissant les pertes par convection interne,

b : constante associée aux pertes par convection externe pour 1 vent de $3 \mathrm{~m} / \mathrm{s}$,

$\varepsilon_{1} \quad$ : emissivité fictive de la vitre externe pour le rayonnement terrestre par ciel clair (cf. [2]).

La résolution numérique nécessite l'emploi du calcul automatique. Elle conduit au résultat consigné sur la figure 3. Nous y lisons toutes les espérances livrées par un rayonnement d'intensité suffisante mais également les inéluctables limitations liées aux faibles éclairements ceci d'autant plus que s'abaisse la température ambiante.
2.2 THERMOCINÉTIQUE. - Les résultats précédents n'ont de sens que pour un récepteur de valeur en eau fixe et un rayonnement incident donné de durée indéfinie. Or dans la réalité l'insolation efficace ne dure que quelques heures. Il convient donc d'optimiser le collecteur pour qu'il puisse atteindre en un temps limité une température suffisante.

Sans revenir sur l'étude complète du problème traité ailleurs [2] posons simplement que la vitesse de chauffe est proportionnelle à la différence entre $T_{\mathrm{e}}$ température limite associée à $G$ et $T$ température actuelle du collecteur et inversement proportionnelle à $M$ valeur en eau par unité de surface captante.

$\left(M\right.$ en $\mathrm{kcal} / U, M=m_{\text {collecteur }} \cdot C+m_{\text {eau }}$,

$C$ en $\mathrm{kcal} / \mathrm{kg}, m$ en $\mathrm{kg} / \mathrm{m}^{2}$ )

$$
\frac{\mathrm{d} T}{\mathrm{~d} t}=\frac{k}{M}\left(T_{\mathrm{e}}-T\right)
$$




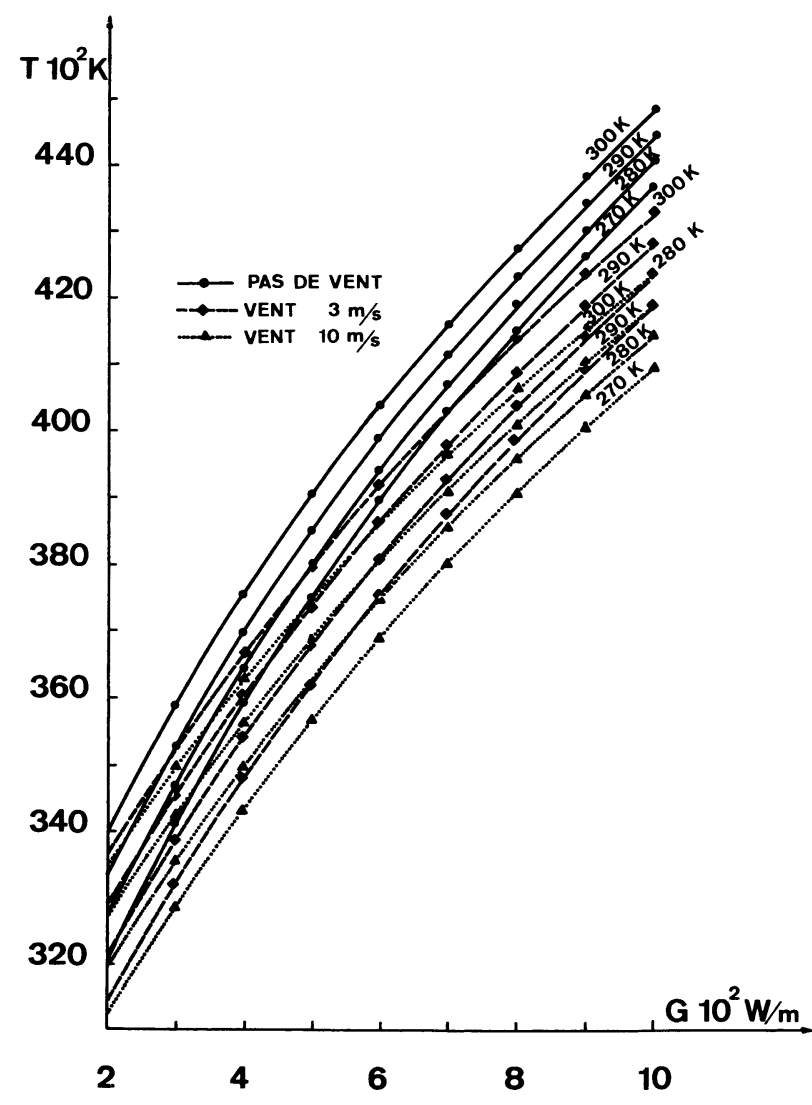

FIG. 3. - Température d'équilibre d'un insolateur (2 vitres, corps noir) en fonction du rayonnement global $G$.

[Equilibrium temperature of a flat collector ( 2 glasses), black body versus global radiation.]

soit :

$$
\left(T_{\mathrm{e}}-T\right)=\left(T_{\mathrm{e}}-T_{0}\right) \exp \left(-\frac{k}{M} t\right)
$$

Nous sommes donc certains, dans une région donnée, de ne pouvoir atteindre $T_{\mathrm{e}}$ défini par $G$ et nous chaufferons d'autant moins vite le fluide caloporteur que $M$ est grand.

Nous aboutissons ainsi à une troisième conclusion essentielle : en augmentant les surfaces collectrices nous pourrons toujours réduire $M$ à une valeur compatible avec la durée d'insolation efficace mais la température maximale du fluide est rigoureusement déterminée par l'éclairement incident. On songe à la réflexion désabusée de Lord Kelvin : les milliards de calories présentes dans l'océan ne pourront jamais faire cuire un œuf. L'inégalité règne même parmi les quantités de chaleur. Or pour chauffer une maison nous avons besoin de calories nobles, c'est-à-dire correspondant à une température suffisamment élevée. Cela est envisageable, dans nos régions, à la demisaison. Ce ne le sera qu'exceptionnellement en hiver.

2.3 Possibilités et Besolns. - Possibilités. - Dans la perspective du chauffage solaire domestique à base de capteurs plans évaluons la surface réceptrice possible. Nous retenons cette solution car elle permet de conserver de larges baies au sud de la construction. Il est nécessaire dans nos régions de rechercher le maximum de clarté et de recevoir le direct lorsque le ciel est dégagé. Elle conduit en outre à une indépendance nécessaire entre le captage et la climatisation de l'habitation. Il ne suffit pas de recevoir l'énergie, il faut pouvoir s'en affranchir l'été. Nous référant aux solutions retenues par E. D. F. à Aramon [5] l'architecte M. Chouleur travaille avec $30 \mathrm{~m}^{2}$ pour le type solaire partiel et $45 \mathrm{~m}^{2}$ pour le type solaire intégral. Il nous paraît difficile d'œuvrer sur plus de $50 \mathrm{~m}^{2}$ répartis sur la toiture et le mur sud, ceci d'autant qu'il convient de conserver une architecture supportable.

Cette évaluation nous apporte en hiver un maximum de $75 \mathrm{kWh}$ incident, $150 \mathrm{kWh}$ à la demi-saison, ce qui nous donne la moitié en admettant un rendement optimiste [6]. Supposons $40 \mathrm{kWh}$ et $80 \mathrm{kWh}$ récupérés. Nous laissons volontairement de côté le problème du potentiel thermique des calories.

- Besoins. - En Lorraine une habitation moyenne 4 pièces cuisine, sanitaires consomme en moyenne $20 \mathrm{~m}^{3}$ de gaz par 24 heures dans une ambiante extérieure de $\left(-10^{\circ} \mathrm{C}\right)$ pour une température intérieure $\mathrm{de}+18^{\circ} \mathrm{C}$ - expérience réellement faite - soit 160 thermies c'est-à-dire $160 \times 1,16=185 \mathrm{kWh}$. On peut en récupérer presque le $1 / 4$ à condition de rendre les calories chauffantes. A la demi-saison environ $100 \mathrm{kWh}$ seront nécessaires, on les couvrira à $80 \%$, la température atteinte par le fluide étant par ailleurs plus élevée. Reste le problème de l'augmentation du potentiel thermique du fluide et de la fluctuation journalière de l'énergie solaire. On le résoudra, au moins en principe, par un réservoir de stockage isolé d'environ $10 \mathrm{~m}^{3}$ d'eau - Aramon $6 \mathrm{~m}^{3}$ pour l'intégral - jouant le rôle de tampon et de source froide, et par l'emploi d'une pompe de chaleur.

3. Conclusion. - Tout en appréciant l'économie qu'est susceptible d'apporter l'emploi de l'énergie solaire pour le chauffage domestique, il convient de rester prudent lorsqu'on fait dans ce domaine de la prospective. En Belgique et en Lorraine :

- le déficit énergétique reste important en hiver (de Novembre à la mi-février),

- les calories récupérées à cette saison sont toujours à bas potentiel thermique, l'emploi d'une pompe de chaleur s'avère alors nécessaire,

- la demi-saison permet un certain optimisme,

- la belle saison impose la limitation à la production d'eau chaude la pompe de chaleur devrait alors permettre une climatisation. La question demeure de sa fiabilité et de son prix.

Le chauffage solaire est gratuit. L'installer coûte cher. 


\section{Bibliographie}

[1] Flechon, J., Martin, G., Toure, M., C. R. Hebd. Séan. Acad. Sci. B 280 (1975) 211-14.

[2] Flechon, J., Revue Phys. Appl. 11 (1976) 743-50.

[3] Duffie, J. A., Beckman, W. A., Solar Energy Thermal Processes (John Wiley) 1974.

[4] Flechon, J., Wertwijn, R., Diallo, A., C. R. Hebd. Séan. Acad. Sci. B 231 (1975) 9-12.

[5] Chouleur, G. : Note 1) sur 5 villas à chauffage avec apport solaire, 2) et 3 villas à chauffage solaire intégral.

[6] Flechon, J., Bull. Union Physiciens 586 (1976) 1307-25. 